\title{
OFF-DIAGONAL LONG-RANGE ORDER IN MANY-ELECTRON PROBLEM
}

\author{
J. Szeftel \\ Laboratoire Léon Brillouin (CEA-CNRS), CE-Saclay \\ 91191 Gif-sur-Yvette Cédex, France
}

The interacting electron Hamiltonian $H=H_{\mathrm{D}}+\sum_{K, \zeta} H_{K, \zeta}$ is considered in the Hilbert space spanned by Slater determinants of Bloch wave functions. $H_{\mathrm{D}}$ consists of the diagonal part of $H$ in this basis. $K$ and $\zeta=0, \pm 1$ stand for the total momentum and projected spin of electron pairs and $H_{K, \zeta}$ is the off-diagonal part of $H$ describing the most general two-electron scattering process conserving $K$ and $\zeta$. It is shown that the eigenspectrum of $H$ includes all eigenvalues of $H_{\mathrm{D}}+H_{K, \zeta}$ for every $K$ and $\zeta$ value. The associated eigenvectors of $H$ are shown to have off-diagonal long-range order.

PACS numbers: 03.65.-w, 71.45.-d, 74.20.-z

\section{Introduction}

The interest for the study of electron correlations in metals has kept growing because of their significance in magnetism and superconductivity $[1,2]$. This work presents a mathematical proof that $H$, a general many-body Hamiltonian, operating in $S_{\phi}$, the Hilbert space spanned by Slater determinants, has numerous eigenstates characterised by having off-diagonal long-range order [3] which was introduced as a fingerprint of the BCS state [2]. To work out the proof, it is necessary to introduce an auxiliary Hilbert space $S_{\otimes \phi}$ which is built over a set of pairs characterised by their total momentum $K$ and projected spin $\zeta$.

\section{The many-body Hamiltonian}

A crystal of arbitrary dimension, containing $N$ sites and $2 n$ electrons where $N \gg 1$ and $n \gg 1$ is considered hereafter. These electrons populate a single band of dispersion $E(k)$ where $k$ is a vector of the Brillouin zone. $E(k)$ is assumed to be independent of the electron spin $\sigma= \pm 1 / 2$. The Pauli principle requires that $n \leq N$. The total system Hamiltonian $H$ can be written in reciprocal space as

$$
H=\sum_{k, \sigma} \dot{E}(k) c_{k, \sigma}^{+} c_{k, \sigma}+\sum_{K, k, k^{\prime}, \sigma_{i=1, \ldots, 4}} V\left(K, k, k^{\prime}\right) c_{k, \sigma_{1}}^{+} c_{K-k, \sigma_{2}}^{+} c_{K-k^{\prime}, \sigma_{3}} c_{k^{\prime}, \sigma_{4}} .
$$

The Fermi operators $c_{k, \sigma}^{+}$and $c_{k, \sigma}$ account for electron creation and annihilation on the Bloch state $k, \sigma$. The real coefficients $V\left(K, k, k^{\prime}\right)$ are the matrix elements 
of the two-electron scattering process. The summations in Eq. (1) are carried out over all possible values of $K, k, k^{\prime}$ in the Brillouin zone under the constraint of spin conservation $\sigma_{1}+\sigma_{2}=\sigma_{3}+\sigma_{4}$. The Hamiltonian $H$ describes the electron motion in the Hilbert space $S_{\phi}$ of dimension $d_{\phi}$. Each basis vector $\phi_{i}$ with $i=1 \ldots d_{\phi}$ is a Slater determinant involving $2 n$ one-electron Bloch states.

It is convenient to introduce the pair creation and annihilation operators $b_{ \pm 1}^{+}\left(k, k^{\prime}\right)=c_{k, \pm \sigma}^{+} c_{k^{\prime}, \pm \sigma}^{+}, b_{ \pm 1}\left(k, k^{\prime}\right)=c_{k^{\prime}, \pm \sigma} c_{k, \pm \sigma}, b_{0}^{+}\left(k, k^{\prime}\right)=c_{k, \sigma}^{+} c_{k^{\prime},-\sigma}^{+}, b_{0}\left(k, k^{\prime}\right)$ $=c_{k^{\prime},-\sigma} c_{k, \sigma}$. The subscript $\zeta=0, \pm 1$ stands for the projection of the total spin of the pair. It is useful to recast the Hamiltonian $H$ of Eq. (1) in terms of the subsidiary Hamiltonians $H_{\mathrm{D}}, H_{K, \zeta}$ as $H=H_{\mathrm{D}}+\sum_{K, \zeta=0, \pm 1} H_{K, \zeta}$ where $H_{\mathrm{D}}$ and $H_{K, \zeta}$ read as

$$
\begin{aligned}
H_{\mathrm{D}} & =\sum_{k, \sigma} E(k) c_{k, \sigma}^{+} c_{k, \sigma}+\sum_{k, k^{\prime}} V\left(k+k^{\prime}, k, k\right) c_{k, \sigma}^{+} c_{k, \sigma} c_{k^{\prime},-\sigma}^{+} c_{k^{\prime},-\sigma} \\
& +\sum_{k, k^{\prime}, \sigma}\left[V\left(k+k^{\prime}, k, k\right)-V\left(k+k^{\prime}, k, k^{\prime}\right)\right] c_{k, \sigma}^{+} c_{k, \sigma} c_{k^{\prime}, \sigma}^{+} c_{k^{\prime}, \sigma}, \\
H_{K, 0} & =\sum_{k, k^{\prime} \neq k} V\left(K, k, k^{\prime}\right) b_{0}^{+}(k, K-k) b_{0}\left(k^{\prime}, K-k^{\prime}\right), \\
H_{K, \pm 1} & =\sum_{k, k^{\prime} \neq(k, K-k)} V\left(K, k, k^{\prime}\right) b_{ \pm 1}^{+}(k, K-k) b_{ \pm 1}\left(k^{\prime}, K-k^{\prime}\right) .
\end{aligned}
$$

The purpose of this article is to demonstrate the following theorem characterising a class of eigensolutions $\psi, \epsilon$ of the Schrödinger equation $(H-\epsilon) \psi=0$ where $H$ is given by Eq. (1) and $\psi$ belongs to the Hilbert space $S_{\phi}$ :

\section{Theorem}

To each eigensolution $\psi_{K, \zeta}, \epsilon$ where $\left(H_{\mathbf{D}}+H_{K, \zeta}-\epsilon\right) \psi_{K, \zeta}=0$, there corresponds an eigensolution $\psi, \epsilon$ of $H$ such that $(H-\epsilon) \psi=0$.

Furthermore it will be shown that $\psi$ has off-diagonal long-range order. Although it is easy to prove [4] this theorem in $S_{\phi}$ for a single pair $(n=1)$, it becomes necessary to treat the problem in an auxiliary Hilbert space $[5,6] S_{\otimes \phi}$ for $n>1$.

\section{Properties of $S_{\otimes \phi}$}

Any Slater determinant $\phi_{i}$ of $S_{\phi}$ can be written as

$$
\phi_{i}=\prod_{K, \zeta}\left(\prod_{j=1}^{n_{K, \zeta}} b_{\zeta}^{+}\left(k_{j}, K-k_{j}\right)\right)|0\rangle,
$$

where $|0\rangle$ designates the no-electron state and all pairs $b_{\zeta}^{+}\left(k_{j}, K-k_{j}\right)|0\rangle$ having the same $K$ and $\zeta$ have been regrouped together. In the product with respect to the index $j$, the $i$ dependence of $j$ has been dropped for simplicity. The integer $n_{K, \zeta} \geq 0$ designates the total number of pairs characterised by $K, \zeta$ in $\phi_{i}$, and the $n_{K, \zeta}$ 's satisfy $\sum_{K, \zeta} n_{K, \zeta}=n$. The basis vector $\Phi_{i, \alpha}$ of $S_{\otimes \phi}$ is defined from $\phi_{i}$ as

$$
\Phi_{i, \alpha}=\bigotimes_{K, \zeta} \phi_{K, \zeta}, \quad \phi_{K, \zeta}=\prod_{i=1}^{n_{K, \zeta}} b_{\zeta}^{+}\left(k_{j}, K-k_{j}\right)|0\rangle,
$$


where the tensor product replaces the simple product $\prod_{K, \zeta}$ of Eq. (3) and each $\phi_{K, \zeta}$ is a Slater determinant containing $n_{K, \zeta}$ of pairs $K, \zeta$. The sequence of integers $\left\{n_{K, \zeta}\right\}$ in Eqs. (3), (4) defines uniquely the pair configuration $\alpha$ of $\phi_{i}$. As a large number of linearly independent vectors $\Phi_{i, \alpha} \in S_{\otimes \phi}$ are characterised by the same pair configuration $\alpha, n_{K, \zeta}$ does not depend on the index $i$ but conversely depends on the index $\alpha$ and will therefore be denoted $n_{K, \zeta, \alpha}$ in the following. The whole set of pair configurations of $\phi_{i}$ is obtained by selecting $m$ permutations of $2 n$ one-electron Bloch states defining $\phi_{i}$. The basis vectors $\Phi_{i, \alpha}$ of $S_{\otimes \phi}$ are generated by allowing the subscripts $i=1 \ldots d_{\phi}$ and $\alpha=1 \ldots m$ to run over all possible values, which implies that the dimension of $S_{\otimes \phi}$ is equal to $m d_{\phi}$. The $\Phi_{i, \alpha}$ 's are chosen to be orthonormal.

The subspace $S_{\Phi} \subset S_{\otimes \phi}$ is then introduced as spanned by the basis vectors $\Phi_{i}$ defined by

$$
\Phi_{i}=\sum_{\alpha=1}^{m} \Phi_{i, \alpha},
$$

where the sum is carried over $m$ pair configurations $\alpha$ of $\phi_{i}$. Owing to the one to one correspondence between $\phi_{i} \in S_{\phi}$ and $\Phi_{i} \in S_{\Phi}$, the dimension of $S_{\Phi}$ is inferred to be equal to $d_{\phi}$.

Introduce now the subspaces $S_{K, \zeta} \subset S_{\Phi}$ and $S_{2} \subset S_{\Phi}$, where $S_{K, \zeta}$ is defined for each $K, \zeta$ as spanned by the basis vectors $\Phi_{i=1 \ldots d_{\zeta}}, d_{\zeta}$ being the dimension of $S_{K, \zeta}$. By definition each $\Phi_{i}$ is associated with a Slater determinant of $S_{\phi}$, comprising $n$ pairs, all having the same $K$ and $\zeta$. Hence the characteristic property of each $\Phi_{i}$ is that its pair configuration expansion, as given in Eq. (5), involves a particular value $\gamma$ so that the tensor product yielding $\Phi_{i, \gamma}$ as in $\mathrm{Eq}$. (4) reduces to a single Slater determinant $\phi_{K, \zeta}$ containing $n$ of pairs $K, \zeta$. Consequently every number of pairs $K^{\prime}, \zeta^{\prime}$ in $\Phi_{i, \gamma}$ where $K^{\prime}$ and $\zeta^{\prime}$ take all possible values different from $K$ and $\zeta$ respectively, vanish for every $\Phi_{i, \gamma}$. Inversely the subspace $S_{2}$ is spanned by the basis vectors $\Phi_{p=1 \ldots d_{2}}$ of $S_{\Phi}, d_{2}$ being the dimension of $S_{2}$. Each $\Phi_{p}$ is characterised by $n_{K, \zeta, \beta}<n$ for every $K, \zeta, \beta$ value where $\beta$ is the pair configuration index of $\Phi_{p}$ and $n_{K, \zeta, \beta}$ stands for the number of pairs $K, \zeta$ in $\Phi_{p, \beta}$. As the subspaces $S_{2}$ and $S_{K, \zeta}$ are disjoint, they provide a basis for $S_{\Phi}$. $S_{\otimes \phi}:$

Consider now the following expression for the Hamiltonian $H^{\prime}$ operating in

$$
H^{\prime}=\sum_{i, j}\left\langle\phi_{i}|H| \phi_{j}\right\rangle\left|\Phi_{i, \gamma}\right\rangle\left\langle\Phi_{j, \gamma}\left|+\sum_{p, q, \beta} m_{p q}\left\langle\phi_{p}|H| \phi_{q}\right\rangle\right| \Phi_{p, \beta}\right\rangle\left\langle\Phi_{q, \beta}\right|,
$$

where the sum with respect to $i, j$ is performed on all Slater determinants $\phi_{i}$ and $\phi_{j}$ associated respectively with $\Phi_{i} \in S_{K, \zeta}$ and $\Phi_{j} \in S_{K, \zeta}$ characterised by the pair configuration $\gamma$. The sum with respect to $p, q$ is carried over all $\Phi_{p}$ and $\Phi_{q}$ such that $\Phi_{p}$ or $\Phi_{q}$ belong to $S_{2}$. The sum with respect to $\beta$ is made with $m_{p p}=1 / m$ and $m_{p q}=(2 n-1) / m$ over all pair configurations common to $\Phi_{p}$ and $\Phi_{q}$. This definition of $H^{\prime}$ in Eq. (6) ensures that the matrix elements $\left\langle\Phi_{e}\left|H^{\prime}\right| \Phi_{f}\right\rangle$, where $H^{\prime}$ is given by Eq. (6), and $\left\langle\phi_{e}|H| \phi_{f}\right\rangle$, where $H$ is given by Eq. (1), are equal for all $e, f=1 \ldots d_{\phi}$ values where $\phi_{e}, \phi_{f}$ are two Slater determinants of $S_{\phi}$ and $\Phi_{e}, \Phi_{f}$ are the corresponding basis vectors of $S_{\Phi}$. This ensures that the Schrödinger 
equations $(H-\epsilon) \psi=0$ and $\left(H^{\prime}-\epsilon\right) \Psi=0$, where $\psi \in S_{\phi}$ and $\Psi \in S_{\Phi}$, have the same spectrum of eigenvalues $\epsilon$.

Since $H^{\prime}$ in Eq. (6) does not display such terms as $\left|\Phi_{p, \alpha}\right\rangle\left\langle\Phi_{q, \beta}\right|$ which would mix two different pair configurations $\alpha$ and $\beta$, the Schrödinger equation $\left(H^{\prime}-\epsilon\right) \Psi=0$ splits into partial Schrödinger equations

$$
\begin{aligned}
& \left(H^{\prime}-\epsilon\right) \Psi=0, \quad \Psi=\sum_{e=1}^{d_{\phi}} a_{e} \Phi_{e}, \quad \Phi_{e}=\sum_{\alpha=1}^{m} \Phi_{e, \alpha} \Rightarrow\left(H^{\prime}-\epsilon\right) \Psi_{\alpha}=0 \\
& \Psi_{\alpha}=\sum_{e=1}^{d_{\phi}} a_{e} \Phi_{e, \alpha}, \quad \Psi=\sum_{\alpha} \Psi_{\alpha}
\end{aligned}
$$

where the coefficients $a_{e}$ are real, the sum over $\alpha$ is the pair configuration expansion of $\Phi_{e}$, and $\Psi_{\alpha}$ belongs to $S_{\otimes \phi}$.

\section{Proof of the theorem}

Consider the Schrödinger equation $\left(H^{\prime}-\epsilon\right) \Psi=0$ where $H^{\prime}$ is given by Eq. (6) and the eigenvector $\Psi \in S_{\Phi}$ is assumed to have a non-vanishing projection in $S_{K, \zeta}$ and thus reads

$$
\Psi=\Psi_{K, \zeta}+\Psi^{\prime}, \quad \Psi_{K, \zeta}=\sum_{i=1}^{d_{\zeta}} a_{i} \Phi_{i}, \quad \Psi^{\prime}=\sum_{p=1}^{d_{2}} a_{p} \Phi_{p},
$$

where the coefficients $a_{i}, a_{p}$ are real and the $\Phi_{i}$ 's and $\Phi_{p}$ 's are basis vectors of $S_{K, \zeta}$ and $S_{2}$, respectively. We now apply Eq. (7) to $\Psi$ for the particular pair configuration $\gamma$ :

$$
\left(H^{\prime}-\epsilon\right) \Psi_{\gamma}=0, \quad \Psi_{\gamma}=\Psi_{K, \zeta, \gamma}+\Psi_{\gamma}^{\prime}
$$

As the vector $\Psi^{\prime}$ is inferred from its definition not to contribute to $\Psi_{\gamma}$, it ensues that $\Psi_{\gamma}$ reduces to $\Psi_{K, \zeta, \gamma}$. Because of $\left\langle\phi_{i}|H| \phi_{j}\right\rangle=\left\langle\phi_{i}\left|H_{\mathrm{D}}+H_{K, \zeta}\right| \phi_{j}\right\rangle$ which holds for the Hamiltonians $H_{\mathrm{D}}$ and $H_{K, \zeta}$ in Eq. (2) and any two Slater determinants $\phi_{i}, \phi_{j}$ associated with the basis vectors $\Phi_{i}, \Phi_{j}$ of $S_{K, \zeta}$, it comes finally

$$
\begin{gathered}
\left(H^{\prime}-\epsilon\right) \Psi_{\gamma}=0 \Rightarrow\left(H_{\mathrm{D}}+H_{K, \zeta}-\epsilon\right) \Psi_{K, \zeta, \gamma}=0 \\
\Leftrightarrow\left(H_{\mathrm{D}}+H_{K, \zeta}-\epsilon\right) \psi_{K, \zeta}=0,
\end{gathered}
$$

where $\psi_{K, \zeta} \in S_{\phi}$ is in one to one correspondence with $\Psi_{K, \zeta} \in S_{\Phi}$. Equation (10) means that if $\left(\psi_{K, \zeta}+\psi^{\prime}\right)$ and $\epsilon$ are eigenvector and eigenvalue of $H$ in $S_{\phi}$, the vector $\psi_{K, \zeta}$ and $\epsilon$ are eigenvector and eigenvalue of $\left(H_{\mathrm{D}}+H_{K, \zeta}\right)$ in $S_{\phi}$ too. To complete the proof of theorem it must be shown in addition that every eigensolution $\psi_{K, \zeta}, \epsilon$ of $\left(H_{\mathrm{D}}+H_{K, \zeta}\right)$ gives rise to an eigensolution $\psi, \epsilon$ of $H$. The latter will be proved now by contradiction. Suppose that there is an eigenvalue of some Hamiltonian $\left(H_{\mathrm{D}}+H_{K, \zeta}\right)$ which is not an eigenvalue of $H$. Then the corresponding $S_{K, \zeta}$ will contribute only $\left(d_{\zeta}-1\right)$ eigenvalues instead of $d_{\zeta}$ to the spectrum of $H$, which will result in an uncomplete diagonal basis for $H$ and is thus at odds with the property of $H$ being hermitian. Q.E.D.

Because $\psi_{K, \zeta}$ and the BCS variational state [2] consist both of a linear combination of Slater determinants of pairs having the same $K, \zeta$, they are characterised by off-diagonal long-range order [3]:

$$
f_{\text {odlro }}(|\tau|)=\sum_{i, j, l, m, \eta}\left\langle\phi\left|c_{i, \sigma}^{+} c_{j, \eta \sigma}^{+} c_{m, \eta \sigma} c_{l, \sigma}\right| \phi\right\rangle,
$$


where $\eta= \pm 1$, the Wannier operator $c_{i, \sigma}^{(+)}$destroys (creates) an electron with spin $\sigma$ at site $i$ labeled by the lattice vector $r_{i},\left(r_{j}-r_{i}\right)=\left(r_{m}-r_{l}\right)=\rho,\left(r_{i}-r_{l}\right)=\tau$. The two-body correlation function $f_{\text {odlro }}(|\tau|)$ is calculated at $\rho$ kept fixed. The state $\phi \in S_{\phi}$ is said to have off-diagonal long-range order if $f_{\text {odlro }}(|\tau|)$ oscillates without decaying to zero for $|\tau| \rightarrow \infty$. Because for $\psi_{K, \zeta}$ and the BCS state it comes $f_{\text {odlro }}(|\tau|)=\cos (K \tau) \Delta$ where $\Delta=\sum_{k, k^{\prime}} \cos \left[\left(k-k^{\prime}\right) \rho\right]\left(b_{\zeta}^{+}(k, K-k) b_{\zeta}\left(k^{\prime}, K-k^{\prime}\right)\right\rangle$, these both states are seen to have off-diagonal long-range order provided $\Delta \neq 0$.

\section{Conclusion}

The conclusion of the theorem is valid for arbitrary crystal dimension, electron concentration and two-electron coupling provided it conserves $K$ and $\zeta$. It enables one to find out all eigenstates of $H$ having off-diagonal long-range order on a cluster of size considerably larger than currently reached, because the dimension of $S_{K, \zeta}$ is much smaller than that of $S_{\phi}$.

I dedicate this work to the memory of my parents Jochweta and Chaim and my niece Denise Lévy and I thank my wife Rachel and children Jérémie and Judith for providing encouragement.

\section{References}

[1] M.W. Long, Int. J. Mod. Phys. B 5, 865 (1991).

[2] J. Bardeen, L.N. Cooper, J.R. Schrieffer, Phys. Rev. 108, 1175 (1957).

[3] C.N. Yang, Phys. Rev. Lett. 63, 2144 (1989).

[4] J. Hubbard, Proc. R. Soc. A 276, 238 (1963).

[5] J. Szeftel, Acta Phys. Pol. A 85, 329 (1994).

[6] J. Szeftel, Physica B 206-207, 705 (1995). 